\title{
PRONOSTICO
}

\section{Los factores de riesgo para padecer diabetes gestacional son similares a los de la diabetes no insulino dependiente}

A Prospective Study of Pregravid Determinants of Gestational Diabetes Mellitus. Solomon CG, Willet WC, Carey VJ. JAMA 1997; 278: 1078-83.

\section{Objetivo}

Evaluar si los factores de riesgo conocidos de diabetes no insulino dependiente (DNID) son determinantes de riesgo de diabetes mellitus gestacional (DMG).

\section{Diseño}

Estudio de cohorte prospectivo por medio de cuestionarios enviados por correo. Estudio de salud de las enfermeras II.

Lugar

14 estados de EE.UU.

Pacientes

Se incluyeron 14.613 enfermeras de 24 a 42 años de edad, sin
DMG previa ni otro tipo de diabetes conocida que reportaron un embarazo único entre 1990 y 1994. De ellas, 722 (4,9\%) reportaron un nuevo diagnóstico de DMG.

\section{Evaluación de factores pronósticos}

Se evaluaron los datos autoreportados sobre edad, historia familiar de diabetes, raza, índice de masa corporal (IMC), cambio de peso desde los 18 años hasta el cuestionario basal en 1989 y tabaquismo activo o pasado. La validez de los datos autoreportados fue comprobada en un subgrupo elegido al azar.

\section{Medición de resultados principales}

Para cada factor de riesgo se calcularon los riesgos relativos e intervalos de confianza de padecer DMG ajustados por edad y otros potenciales confundidores.

\section{Resultados principales}

El riesgo de DMG aumentó significativamente con:

\begin{tabular}{|c|c|c|c|}
\hline \multicolumn{2}{|c|}{ 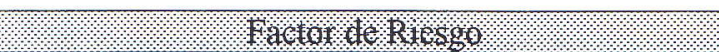 } & (1) & $1(1.08 \%$ \\
\hline 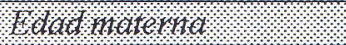 & $\geq 40$ años vs. $25-29$ años & 2.38 & $1.26-3.98$ \\
\hline \multirow{2}{*}{ 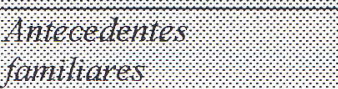 } & Madre & 1.90 & $1.49-2.42$ \\
\hline & Ambos progenitores & 2.87 & $1.48-5.58$ \\
\hline \multirow{2}{*}{ 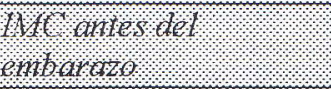 } & $25-29,9$ vs. $<20$ & 2.13 & $1.65-2.74$ \\
\hline & $>30$ vs. $<20$ & 2.9 & $2.15-3.91$ \\
\hline \multirow{3}{*}{ 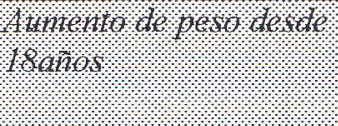 } & $5-9.9 \mathrm{~kg}$. & 1.67 & $1.37-2.05$ \\
\hline & $10-19.9 \mathrm{~kg}$. & 2.54 & $2.07-3.12$ \\
\hline & $\geq 20 \mathrm{~kg}$ & 3.56 & $2.70-4.69$ \\
\hline THW H & & 1.43 & $1.14-1.80$ \\
\hline 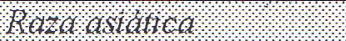 & & 2.32 & $1.52-3.54$ \\
\hline
\end{tabular}

No se observó una diferencia significativa del riesgo en mujeres que hacían ejercicios vigorosos antes del embarazo comparadas con las sedentarias.

\section{Conclusiones}

La presencia de los reconocidos factores de riesgo de diabetes no insulino dependiente aumenta también el riesgo de diabetes gestacional.

Fuente de financiamiento: No referida.

\section{Comentario}

Este es un excelente trabajo para demostrar lo que se propone. Las mujeres que tienen 40 años o más, historia parental de diabetes, raza asiática, IMC pregrávido > de 25 o aumento de peso de más de $5 \mathrm{~kg}$ desde los 18 años, y el fumar antes del embarazo aumentan la probabilidad de diabetes gestacional. Es interesante destacar que el 60\% de las mujeres con DMG de esta cohorte (prevalencia de DMG de 4,9\% y similar a la reportada en la literatura) no presentan ninguno de estos factores de riesgo. Esto no hace más que reafirmar la controversia de la indicación de rastreo universal de DMG a toda mujer embarazada (recomendación tipo $\left.C^{*}\right)(1)$. De todas formas creemos que los médicos de atención primaria debemos estar familiarizados con estos factores de riesgo asociados al desarrollo de diabetes y aconsejar mantener un peso adecuado y evitar el tabaquismo. Es bueno recordar que la prueba indicada para el rastreo durante el embarazo en pacientes de riesgo es la prueba oral de 50 gramos de glucosa y sólo hacer el test de tolerancia de $100 \mathrm{~g}$ en las que el resultado es anormal. Faltan estudios prospectivos que demuestren el rol beneficioso de la actividad física para la prevención de la DMG.

*Ver glosario

Marcela Botargues Unidad de Medicina Familiar y Preventiva

\section{Referencias}

1. US Preventive Services Task Force. Guide to clinical preventive services, 2ed. Baltimore: Wiliams \& Wilkins, 1996.pp193-208 


\section{La isquemia silente es un predictor de mortalidad coronaria}

\begin{abstract}
Silent ST-T changes in an epidemiologic cohort study. A marker of hypertension or coronary heart disease, or both: The Reykjavilk Study. Sigurdsson E, Sigfusson N, Sigvaldason H, et al. JACC 1997;27:1140-7
\end{abstract}

\section{Objetivos}

1) Describir características clínicas y bioquímicas de un grupo de pacientes portadores de alteraciones del segmento ST-T interpretadas como isquemia silente (IS); 2) Analizar el valor predictor independiente de la IS en relación con: desarrollo de angina de pecho (AP), infarto agudo de miocardio (IAM) y muerte coronaria (MC); 3) Comparar la evolución clínica de los pacientes portadores de IS vs. otras formas clínicas de enfermedad coronaria.

\section{Diseño}

Estudio prospectivo de cohorte.

\section{Lugar}

Reikjavik, Islandia.

\section{Pacientes}

Fueron reclutados 9.139 hombres a partir del año 1.967 con seguimiento hasta el año $1.992(71 \%$ de la población total invitada a participar en el trabajo. Se analizaron los siguientes grupos: 1) Pacientes con alteraciones isquémicas del ST-T en el electrocardiograma (ECG) de reposo, definidas por el código de Minnesota, sin síntomas ni antecedentes coronarios (Grupo IS); 2)Pacientes sin evidencia de isquemia sintomática o silente (Grupo control); y 3) Pacientes con antecedentes AP o IAM (con o sin IS).

\section{Evaluación de factores prónosticos}

Presencia de isquemia silente, edad, intolerancia a la glucosa, colesterolemia, trigliceridemia, uricemia, tensión arterial sistólica (TAS), diastólica, índice de masa corporal (IMC), presencia de hipertrofia ventricular izquierda (HVI) en el ECG, hipertensión con y sin tratamiento, tabaquismo, diuréticos y digoxina.

\section{Medidas de Resultados}

Todos los pacientes fueron evaluados con examen físico, síntomas coronarios, laboratorio, radiografía de tórax y ECG. La cau- sa de muerte se determinó a partir del certificado de defunción y en el $55 \%$ de los casos por autopsia.

\section{Resultados Principales}

1) Comparación de las características del Grupo IS $(n=437)$ vs. el Grupo Control ( $\mathrm{n}=7903)$ :

Las variables asociadas a IS (análisis multivariado) fueron: tratamiento con diuréticos, tratamiento con digital, antihipertersivos cardiomegalia, TAS, edad, cigarrillos $\geq 15 /$ día e HVI. 2) Predicción de eventos coronarios: el tratamiento con diuréticos, digital, la hipercolestérolemia, hipertrigliceridemia y la edad mostraron un valor predictor independiente para AP o IAM. Luego de ajustar por las distintas variables predictoras, la IS se asoció a un riesgo 1.6 veces mayor de eventos ( $R^{*}$ ), pero estos valores no alcanzaron niveles significativos (IC 95\% 1-2.8, p 0.07). La IS mostró valor predictor independiente para muerte de causa coronaria (RR 2.0, IC 95\% 1.6 - 2.5 ). 3) Pronóstico: Las diferentes manifestaciones de enfermedad coronaria analizadas se asociaron a un aumento en el riesgo de muerte coronaria en relación con el grupo control (ver tabla)

\begin{tabular}{|c|c|c|}
\hline Heen & $\mathrm{KH}$ & $1 \mathrm{Q} / 0$ \\
\hline I & 2.0 & $1.6-2.6$ \\
\hline $11 / 511 \%$ & 2.5 & $2.0-3.2$ \\
\hline WI $1011 \mathrm{SS}$ & 4.2 & $3.1-5.7$ \\
\hline WX sin I 1 & 5.6 & $4.0-7.8$ \\
\hline WAN/OOIS & 9.9 & $7.6-13$ \\
\hline
\end{tabular}

\section{Conclusión}

La IS fue un predictor independiente de muerte de causa coronaria, si bien el pronóstico de estos pacientes fue mejor que el de otras formas clínicas de enfermedad coronaria.

triculo izquierdo o tratados con digital en este grupo; 2) El rechazo del $29 \%$ de los pacientes invitados a participar. No se puede determinar si las características de este subgrupo difieren del resto esto modifica o no los resultados obtenidos, comprometiendo la validez externa*. Sus datos más destacables fueron la importante cantidad de pacientes incluidos y la evaluación de mortalidad partir de las autopsias en un gran porcentaje de casos.

*Ver glosario

Dr Alberto Velázquez Unidad de Medicina Familiar y Preventing Hospital Italiano de Buenos Aire

\section{Referencia}

Ruthesford Johnd, Braunwald E., Silent Myocardiot Ischemia, en Hean Discose, W.B. Saunders 1992 pp 1347 - 50 